\title{
ОПРЕДЕЛЕНИЕ ОПТИМАЛЬНЫХ ЗНАЧЕНИЙ ЦЕЛЕВЫХ ИНДЕКСОВ НАДЕЖНОСТИ СТРОИТЕЛЬНЫХ КОНСТРУКЦИЙ ДЛЯ ПЛОСКИХ ПЕРЕКРЫТИЙ КАРКАСНЫХ ЗДАНИЙ
}

\author{
А. И. Лапина ${ }^{1}$ \\ 1 Старший преподаватель кафедры архитектуры учреждения образования \\ «Брестский государственньй технический университет», Брест, Беларусь, e-mail: 375298211966@yandex.by
}

\begin{abstract}
Реферат
Целевые уровни надежности, рекомендуемые в различных национальных и международных документах, противоречивы. В различных источниках они определяются в зависимости от относительной стоимости мер безопасности, последствий отказа, риска для жизни, ставки дисконтирования и т. д. Очевидно, что оптимальные значения индексов надежности для различных конструкций могут сильно отличатся. В данной статье предложена методика определения целевых индексов надежности для безбалочных плит перекрытий каркасных жилых зданий. Эта проблема решена на основе вероятностной оптимизации и критерия принятия риска обществом, который входит в решение в качестве граничного условия. В результате исследования были определены значения индексов надежности для безбалочных плит, отличающихся размером пролета и величиной приложенной нагрузки.
\end{abstract}

Ключевые слова: индекс надежности, плоские перекрытия, оптимизация.

\section{DETERMINING THE OPTIMAL VALUES OF TARGET RELIABILITY INDEX OF BUILDING STRUCTURES FOR FLAT FLOORS OF FRAME BUILDINGS}

\begin{abstract}
Keywords: reliability index, flat slabs, optimization.

\section{Введение}

Любая инженерная задача имеет множество возможных решений. Оптимальное проектирование позволяет определить наилучшее из них по заданному критерию. В качестве такого критерия обычно используются экономические показатели. Очевидно, что выбор наилучшего решения напрямую связан с надежностью конструкции. При этом следует отметить, что наиболее выгодная с экономической точки зрения конструкция не всегда наименее надежна. Это было наглядно проиллюстрированное еще в работе Клаина [1], в который он доказал, что трехстержневая металлическая ферма легче (а значит экономичнее), чем двухстержневая, несмотря на более высокие резервы прочности. Таким образом, для любой конструкции существует свой экономически целесообразный уровень надежности, в соответствии с которым ее и следует проектировать. Так как отказ конструкции влечет за собой риск для жизни людей, надежность конструкций должна быть также оценена с социальной точки зрения. Минимальное значение вероятности отказа конструкции должно быть определен с использованием оценки эффрективность мер по спасению жизни.
\end{abstract}

Target reliability levels recommended in various national and international documents are inconsistent. In various sources, they are determined depending on the relative costs of safety measures, the consequences of failure, the risk to life, discount rate, etc. It is obvious that the optimal values of reliability index for various designs are very different. In the present paper, a method for determining the target reliability index for flat floor slabs of frame buildings is proposed. This problem is solved based on probabilistic optimization and the societal risk acceptance criterion, which enters the decision as a boundary condition. As a result of the research, the values of the reliability index for flat slabs, with different span sizes and applied loads, were determined.

\section{Описание модели исследования}

В настоящей статье сформированы основные положения методики определения оптимальных целевых значений индексов надежности для безбалочных перекрытий каркасных зданий. В качестве модели для решения данной задачи рассмотрим квадратный в плане фрагмент каркаса здания, вырезанный по линиям нулевых поперечных сил в перекрытии (рис. 1).

Высота этажа $\left(H_{\ni}\right)$ принята 3 м, количество этажей $\left(N_{\ni}\right) 10$. Будем рассматривать перекрытия пролетом $(L)$ от 5 до 8 м, загруженные равномерно распределенной нагрузкой. Постоянная часть нагрузки на перекрытие включает в себя собственный вес конструкции, а также вес пола, потолка, сантехнических разводок и т. д. Будем рассматривать конструкции полезная часть постоянной нагрузки на перекрытия $\left(G_{\Pi}\right)$, для которых составляет $2 \mathrm{kH} / \mathrm{M}^{2}$,
$4 \mathrm{kH} / \mathrm{M}^{2}$ и 8 kH/M². В соответствии с CH 2.01.01-2019 [2] постоянная нагрузка имеет нормальное распределение, а коэффициент вариации для нее может быть принят от 0,05 до 0,1.

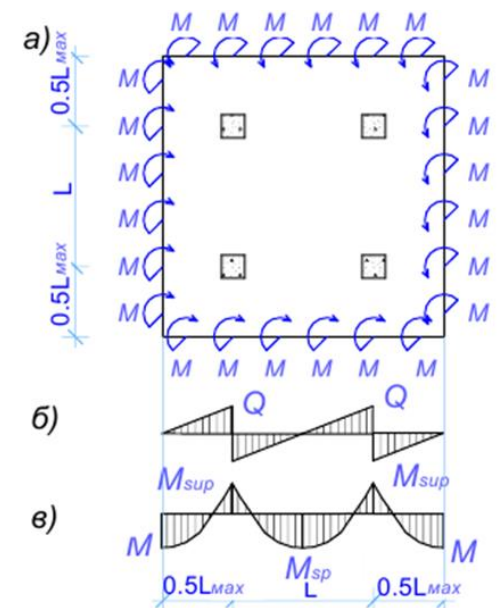

а) ячейка перекрытия, вырезанная по линиям нулевых значений поперечной силы; б) эпюра поперечных сил;

в) эпюра изгибающих моментов

Рисунок 1 - Модель для решения задачи

Кроме собственного веса на перекрытия жилого здания действует функциональная нагрузка от веса людей и оборудования. Нормативное значение функциональной нагрузки $\left(Q_{k}\right)$ согласно $\mathrm{CH}$ 2.01.01-2019 [2] составляет $2 \mathrm{kH} / \mathrm{M}^{2}$. Вероятностное распределение для этого вида нагрузок менее изучено, результаты статический исследований у различных автором существенно отличаются [3]. По этой причине при решении задачи в первом приближении 
примем, что функциональная нагрузка имеет нормальное распределение. Среднее значение и коэфффициент вариации примем согласно рекомендациям JCSS [4] равными $0,6 Q_{k}$ и 0,35 соответственно.

Будем считать, что рассматриваемый фрагмент (рис.1) изготовлен из монолитного бетона с классом прочности С25/30 без предварительного напряжения арматуры. Согласно СТБ EN 206-1-2011 [5] значение прочности бетона распределено по логнормальному закону, значение стандартного отклонения $\left(\sigma_{c}\right)$ которого составляет 5 МПа. Армирование перекрытия выполнено стержневой арматурой класса S500 связанной на месте установки в плоские сетки, распложенные в нижней и верхней части сечения плиты, поперечная арматура в плите отсутствует. Согласно СТБ EN 10080-2011 [6] значение прочности арматуры распределено по логнормальному закону, значение стандартного отклонения $\left(\sigma_{s}\right)$ которого составляет $80 \mathrm{MПа.} \mathrm{Геометрические} \mathrm{парамет-}$ ры конструкции в виду незначительного коэффицциента вариации не будем рассматривать как стохастические. Сведения о базисных переменных, необходимых для решения задачи сводим в таблицу 1.

Таблица 1 - Базисные переменные

\begin{tabular}{|c|c|c|c|c|c|}
\hline $\begin{array}{l}\text { Базисная } \\
\text { перемен- } \\
\text { ная }\end{array}$ & $\begin{array}{c}\text { Cреднее } \\
\text { значение }\end{array}$ & $\begin{array}{l}\text { Стандарт- } \\
\text { ное откло- } \\
\text { нение }\end{array}$ & $\begin{array}{c}\text { Коэфр-т } \\
\text { вариации }\end{array}$ & $\begin{array}{l}\text { Функция } \\
\text { распреде- } \\
\text { ления }\end{array}$ & $\begin{array}{c}\text { Обос- } \\
\text { но- } \\
\text { вание }\end{array}$ \\
\hline $\begin{array}{c}\text { Постоян- } \\
\text { ная } \\
\text { нагрузка, } \\
\mathrm{kH} / \mathrm{M}^{2} \\
\end{array}$ & $\begin{array}{c}G_{m}=h \cdot 9,81 / \\
/ 1000+G_{n} \\
h-\text { толщинаплиты }\end{array}$ & $\sigma_{G}=G_{m} \cdot V_{G}$ & $V_{G}=0,1$ & \begin{tabular}{|c|} 
нормаль- \\
ное рас- \\
пределение \\
Гаусса
\end{tabular} & $\begin{array}{c}\mathrm{CH} \\
2.01 .01- \\
2014 \text { [2] }\end{array}$ \\
\hline $\begin{array}{c}\text { Функциа- } \\
\text { нальная } \\
\text { нагрузка, } \\
\text { кH/m² } \\
\end{array}$ & $Q_{m}=0,6 \cdot Q_{k}$ & $\sigma_{Q}=Q_{m} \cdot V_{Q}$ & $V_{Q}=0,35$ & $\begin{array}{c}\text { нормаль- } \\
\text { ное рас- } \\
\text { пределение } \\
\text { Гаусса }\end{array}$ & $\begin{array}{c}\mathrm{CH} \\
2.01 .01- \\
2014 \text { [2], } \\
\mathrm{JCSS}[4]\end{array}$ \\
\hline $\begin{array}{c}\text { Прочность } \\
\text { бетона на } \\
\text { сжатие, } \\
\text { МПа }\end{array}$ & $f_{c m}=f_{c k}+1,64 \cdot \sigma_{f c}$ & $\sigma_{c}=5$ & $V_{c}=\sigma_{c} / f_{c m}$ & $\begin{array}{c}\text { логнор- } \\
\text { мальное } \\
\text { распреде- } \\
\text { ление }\end{array}$ & \begin{tabular}{|c} 
CTБEN \\
$206-1-$ \\
$2011[4]$
\end{tabular} \\
\hline $\begin{array}{c}\text { Прочность } \\
\text { арматуры, } \\
\text { МПа }\end{array}$ & $f_{s m}=f_{s k}+1,64 \cdot \sigma_{f s}$ & $\sigma_{s}=80$ & $V_{s}=\sigma_{s} / f_{s m}$ & $\begin{array}{c}\text { логнор- } \\
\text { мальное } \\
\text { распреде- } \\
\text { ление }\end{array}$ & $\begin{array}{l}\text { CTE EN } \\
10080- \\
2011[5]\end{array}$ \\
\hline $\begin{array}{c}\text { Геометри- } \\
\text { ческие } \\
\text { парамет- } \\
\text { ры, м }\end{array}$ & $\begin{array}{c}\text { номинальное } \\
\text { значение }\end{array}$ & - & - & - & $\begin{array}{l}\mathrm{CH} \\
2.01 .01 \\
2014[2]\end{array}$ \\
\hline
\end{tabular}

В качестве варьируемых параметров для решения задачи рассмотрим: толщину перекрытия $(h)$, ширину сечения колонны (b), коэффициент армирования перекрытия $(\rho)$. При расчете прочности плиты на изгиб по методу предельного равновесия примем одинаковый коэффициент армирования в пролете и над опорами перекрытия. Шаг табуляции и пределы изменения варьируемых параметров сведены в таблицу 2.

Таблица 2 - Варьируемые параметры

\begin{tabular}{|l|c|c|c|c|}
\hline \multicolumn{1}{|c|}{$\begin{array}{c}\text { Наименование } \\
\text { параметра }\end{array}$} & $\begin{array}{c}\text { Ед. } \\
\text { имз. }\end{array}$ & $\begin{array}{c}\text { Мин. } \\
\text { знач. }\end{array}$ & $\begin{array}{c}\text { Макс. } \\
\text { знач. }\end{array}$ & Шаг \\
\hline Толщина перекрытия, $h$ & м & 0,16 & 0,40 & 0,02 \\
\hline $\begin{array}{l}\text { Ширина сечения } \\
\text { колонны, } b\end{array}$ & м & 0,3 & 0,75 & 0,05 \\
\hline $\begin{array}{l}\text { Коэфффициент армирования } \\
\text { перекрытия, } \rho\end{array}$ & $\%$ & 0,15 & 2,0 & 0,05 \\
\hline
\end{tabular}

В данной конструкции разрушение может наступить в результате превышения следующих предельных состояний:

- исчерпания сопротивления изгибу;

- исчерпания сопротивлению местному срезу (продавливанию).

Таким образом, вероятность отказа перекрытия будет определятся по формуле:

$$
P_{F}=1-\left(1-P_{F 1}\right) \cdot\left(1-P_{F 2}\right),
$$

где $P_{F i}$ - вероятность разрушения, в результате наступления $i$-го предельного состояния, определяемая в соответствии с СТБ ISO 2394-2007 [7] по формуле:

$$
P_{F i}=P\left(g_{i}(X)<0\right),
$$

где $X$ - вектор базисных переменных (табл. 1).

$g_{i}(X)$ - функция $i$-го предельного состояния, определяемая в общем случае:

$$
g_{i}(X)=R_{i}(X)-S_{i}(X),
$$

где $R_{i}(X)$ - фукция сопративления, для $i$-ой формы отказа,

$S_{i}(X)$ - функция воздействия, для $i$-ой формы отказа.

Функция пределльного состояния для сопротивления изгибу:

$$
g_{1}(X)=R_{1}\left(L, f_{c m}, f_{s m}, h, \rho\right)-S_{1}\left(L, G_{m}, Q_{m}, b, h\right)
$$
срезу:

Функция пределльных состояний для сопротивления местному

$$
g_{2}(X)=R_{2}\left(L, f C_{m}, f_{s m}, h, b, \rho\right)-S_{2}\left(L, G_{m}, Q_{m}, b, h\right)
$$

Согласно с СТБ ISO 2394 [7], для нахождения вероятности отказа будем использовать имитационное моделирование Монте-Карло. На основании данного метода построим гистограммы плотности распределения для функций состояний. Число симуляций принимаем равным 200.

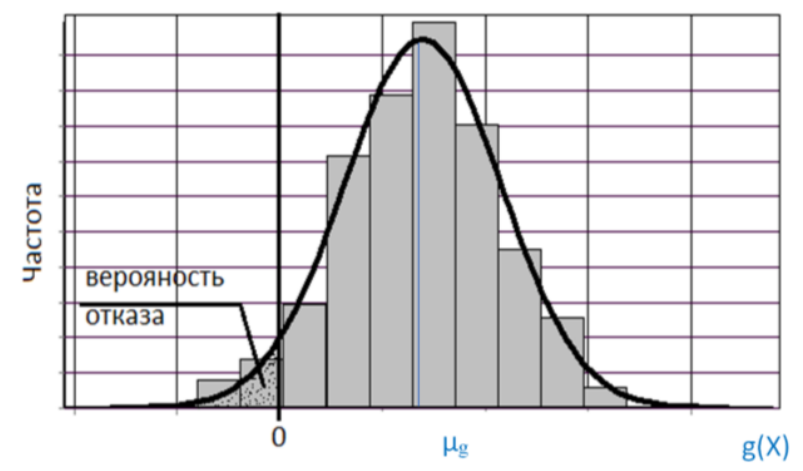

$\square$ гистограмма полученная имметационным
моделированием по методу Монте Карло;
кривая нормального распредления Гаусса

Рисунок 2 - Плотность распределения значений функции состояния

На рисунке 2 приведена гистограмма распределения функции состояния для формы разрушения при изгибе перекрытия пролетом 6 м толщиной $20 \mathrm{~cm}$, опирающейся на колонны сечением 40 × $40 \mathrm{~cm}$. Перекрытие выполнено из бетона с классом прочности С 25/30 и арматуры S500, коэфффициент армирования принят одинаковым для нижней и верхней зоны сечения плиты и составляет $0,15 \%$. Плита загружена собственным весом, постоянной полезной нагрузкой $4 \mathrm{kH} / \mathrm{M}^{2}$, и функциональной нагрузкой $2 \mathrm{kH} / \mathrm{M}^{2}$. Усилия в плите определены методом предельного равновесия, функция сопротивления рассчитана на основании упрощенной деформационной модели.

Полученное распределение для данного случая, как и для всех остальных, близко к нормальному распределению Гаусса, что подтверждается определением критерия Пирсона и внешним видом гистограммы. Параметры полученных распределений определяем по формулам:

$$
\begin{aligned}
& \mu_{g}=\sum g_{i} / N, \\
& \sigma_{g}^{2}=D_{g}=(g i-\bar{g})^{2} /(N-1),
\end{aligned}
$$

где $N$ - количество проведенных численных экспериментов;

$g_{j}-j$-ое значение резерва несущей способности;

$\mu_{g}$ - математическое ожидание резерва несущей способности;

$D_{g}$ - дисперсия резерва несущей способности;

$\sigma_{g}$ - среднеквадратичное отклонение несущей способности.

На основании этих параметров строим кривую нормального распределения резерва прочности и определяем вероятность отказа конструкции, как площадь криволинейной трапеции. Для случая, приведённого на рисунке 2 , она составляет $1,35 \cdot 10^{-2}$ : 


$$
P_{f}=0.5-\Phi\left(\mu_{g} / \sigma_{g}\right),
$$

где $\mu_{g}, \sigma_{g}$ - параметры распределения резерва прочности, определяемые по формулам (6) и (75);

$$
\Phi(\bullet) \text { - функция Лапласа. }
$$

Индекс наждености конструкции будет определятся как обратное значение стандартного нормального интегрального распределения от вероятности отказа конструкции:

$$
\beta=-\Phi^{-1}\left(P_{f}\right),
$$

Для случая, представленного на рисунке 2, индекс надежности составляет 1,4, что соответствует обратному значению стандартного нормального интегрального распределения от вероятности отказа 1,35·10-2.

\section{Постановка задачи оптимального проектирования}

В соответствие с СТБ ISO 2394-2015 [7] целью проектирования считаем минимизацию стоимости конструкций на протяжении всего срока службы, с учетом ликвидации последствий ее вероятностного отказа. Учитывая эти требования и принятую модель (рис. 1), примем в качестве целевой функции общую стоимость плит перекрытия и колонн на протяжении всего срока службы, приведенную на 1 м² эксплуатируемой площади здания, а в качестве критерия оптимальности - минимум этой функ ции.

Так как стоимость конструкции на протяжении всего срока службы включает в себя не только затраты, связанные с возведением, но и затраты, обусловленные износом конструкции, то целевая функции имеет вид:

$$
F=(C(p)+A(p)+D(p)) / S \rightarrow \text { min, }
$$

где $C(p)$ - затраты, связанные с возведением, определяемые по формуле (12);

$A(p)$ - затраты, обусловленные износом, определяемые по формуле (13);

$D(p)$ - затраты, связанные с ликвидацией последствий вероятностного отказа, определяемые по формуле (18);

$p$-варьируемые параметры (табл. 2);

$S$ - эксплуатируемая площадь расчетного участка здания, определяемая по формуле:

$$
S=N \cdot 4\left(L^{2}-b^{2}\right),
$$

где $N$ - количество этажей в здании;

$L$ - шаг колонн, $M$;

$b$ - ширина сечения колонны, $M$.

Затраты, связанные с возведение конструкции, определяем как сметную стоимость $\left(C_{C M P}\right)$ за вычетом плановой прибыли $(П П):$

$$
C(p)=C_{C M P}(p)-\Pi \Pi(p),
$$

Сметную стоимость и плановую прибыль определяем в соответсвии с локальной сметой по инструкции [8]. Виды и объемы работ, необходимые для возведения рассматриваемого фрагмента, указаны в таблице 3.

Таблица 3 - Виды объемы работ для составления локальной сметы

\begin{tabular}{|c|c|c|c|}
\hline $\begin{array}{c}\text { Обосно- } \\
\text { вание }\end{array}$ & Наименование & $\begin{array}{c}\text { Ед. } \\
\text { изм. }\end{array}$ & Кол-во \\
\hline $\begin{array}{c}\text { E6-54-3 } \\
\text { или } \\
\text { E6-54-4 }\end{array}$ & $\begin{array}{c}\text { Бетонирование перекрытий } \\
\text { толинй до } 20 \text { см или свыше } 20 \text { см } \\
\text { в крупнощитовой опалубке }\end{array}$ & $\mathrm{M}^{2}$ & $4 \cdot \mathrm{N} \cdot \mathrm{L} \cdot \mathrm{L}$ \\
\hline E6-57-1 & $\begin{array}{c}\text { Установка арматуры } \\
\text { в перекрытие }\end{array}$ & $\mathrm{T}$ & $\begin{array}{c}4 \cdot \mathrm{N} \cdot 2 \cdot \mathrm{d} \\
\mathrm{L} \cdot \mathrm{L} \cdot \rho \cdot 7,8\end{array}$ \\
\hline $\begin{array}{c}\text { E6-104-1 } \\
\text { или } \\
\text { E6-104-2 }\end{array}$ & $\begin{array}{c}\text { Устройство колонн в опалубке } \\
\text { типа "Модостр" высотой } \\
\text { до 4м, прямоугольных } \\
\text { периметром до 2м или до 3м }\end{array}$ & $\begin{array}{c}100 \\
\mathrm{M}^{3}\end{array}$ & $4 \cdot \mathrm{N} \cdot \mathrm{b} \cdot \mathrm{b} \cdot \mathrm{Hэ} / 100$ \\
\hline
\end{tabular}

При оценке затрат на моральный износ считаем, что конструктивная система систематически обновляется с учетом годового износа $(\omega)$, поэтому:

$$
A(p)=C(p) \cdot w / \gamma,
$$

где $w$ - годовой износ, принимаем для жилых зданий равным $2 \%$;

$Y$ - коэфффициент дисконтирования, согласно [9], принимается в приделах:

$$
\delta \cdot \varepsilon<\gamma<\rho+\delta \cdot \varepsilon,
$$

где $\rho$ - ставка временных предпочтений, принимается равным 3 \% [9];

$\delta$ - темп экономического роста ВВП;

$\varepsilon$ - эластичность потребления, определяется по формуле:

$$
\varepsilon=1-q,
$$

где $q$ - коэффициент, зависящий от продолжительности активной фазы жизни:

$$
q=\omega /(\beta \cdot(1-\omega)),
$$

где $\beta$ - соотношение доходов населения и ВВП, по данным справочников [10-12] за 2010-2019 года для Республики Беларусь колебался в пределах от 0,557 до 0,682 (табл. 4);

$\omega$ - соотношение продолжительности труда и отдыха:

$$
\omega=h \cdot M / P,
$$

где $h$ - отношение количества отработанных часов к общему, с учетом 40-часовой рабочей недели составляет 0,24;

$M / P$ - отношение количества людей, занятых в экономике к общей численности населения, по данным справочников [10-12] за 2010-2020 года это соотношение уменьшилось от 0,49 до 0,46.

Затраты, связанные с ликвидацией последствий вероятностного отказа, определяются по формуле:

$$
D(p)=C_{F}(p) \cdot P_{F}(p),
$$

где $P_{F}(p)$ - вероятность отказа, определяемая по формуле (1);

$C_{F}(p)$ - ущерб от отказа, включающий в себя затраты на реконструкцию или возведение нового здания и затраты, обусловленные наступлением отказа:

$$
C_{f}(p)=(C(p)+H) / \gamma
$$

где $C(p)$ - затраты, связанные с реконструкцией или возведением нового здания, определяемые по формуле (12);

$\gamma$ - коэфффициент дисконтирования, назначается в соответствии с (14);

$H$ - расходы, которые начисляются в случае отказа в дополнение к затратам на реконструкцию, включают в себя затраты на демонтажные работы, а также человеческие жертвы, выраженные в монетарных единицах:

$$
H=C_{\text {ДEM }}+N_{F} \cdot I C A F,
$$

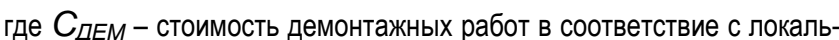
ной сметой, на демонтажные работы, составленной в соответствии с инструкцией [8];

$N_{F}$ - предполагаемое число человеческих жертв, определяемое в зависимости от нормы площади на одного человека, которая для жилых зданий составляет $10 \mathrm{~m}^{2}$ [13]

$I C A F$ - затраты, которую экономически целесообразно инвестировать в сохранение жизни (Implied Cost of Averting a Fatality), зависящая от уровня качества жизни и определяемая в соответствии с нормами СТБ ISO 2394-2015 [7] по формуле:

$$
I C A F=g \cdot e /(4 \cdot q),
$$

где $g$ - валовый внутренний продукт на душу населения;

e- средняя ожидаемая продолжительность жизни;

q - тоже что и в (16).

Расчет ICAF для экономических условий Республики Беларусь приведен в таблице 4, а полученные изменения значений выражения в тысячах долларов США представлены на рисунке 3. 
Вестник Брестского государственного технического университета. 2021

Таблица 4 - Вычисление затрат, которые экономически целесообразно инвестировать в сохранение жизни (ICAF)

в текущих ценах для экономических условий Республики Беларусь за 2010-2019 года

\begin{tabular}{|c|c|c|c|c|c|c|c|c|c|c|c|}
\hline Параметр & Ед. изм. & 2010 & 2011 & 2012 & 2013 & 2014 & 2015 & 2016 & 2017 & 2018 & 2019 \\
\hline $\begin{array}{c}\text { Ожидаемая } \\
\text { продолжительность } \\
\text { жизни при рождении - } \\
e[14-20]\end{array}$ & год & 70,4 & 70,6 & 72,2 & 72,6 & 73,2 & 73,9 & 74,1 & 74,4 & 74,5 & 75 \\
\hline $\mathrm{BB} \sqcap-\mathrm{G}[10-12]$ & млр руб & 16,4 & 29,7 & 53,0 & 64,9 & 77,9 & 89,9 & 94,9 & 105,7 & 122,3 & 132,0 \\
\hline $\mathrm{BB} \sqcap-G[21]$ & млр \$ & 57,22 & 61,37 & 65,67 & 75,50 & 78,74 & 56,33 & 47,70 & 54,72 & 60,01 & 63,09 \\
\hline $\begin{array}{c}\text { Численость } \\
\text { населения - } P \text { [10-12] }\end{array}$ & тыс. чел & 9481 & 9465 & 9464 & 9468 & 9481 & 9498 & 9505 & 9492 & 9475 & 9408 \\
\hline $\begin{array}{l}\text { в том числе занятого } \\
\text { в экономике - M [10-12] }\end{array}$ & тыс. чел & 4666 & 4655 & 4577 & 4546 & 4487 & 4496 & 4406 & 4354 & 4338 & 4330 \\
\hline $\begin{array}{c}\text { Денежные доходы } \\
\text { на душу населния } \\
\text { в месяц - } W[10-12]\end{array}$ & руб. & 95,2 & 145,8 & 283,1 & 389,4 & 459,2 & 494,3 & 514,9 & 562,4 & 639,6 & 718,7 \\
\hline \multirow{2}{*}{$g=G / P$} & руб. & 1735 & 3140 & 5605 & 6856 & 8211 & 9466 & 9985 & 11136 & 12907 & 14030 \\
\hline & $\$$ & 6035 & 6484 & 6939 & 7974 & 8305 & 5930 & 5019 & 5765 & 6333 & 6706 \\
\hline$\beta=W \cdot 12 / g$ & & 0,659 & 0,557 & 0,606 & 0,682 & 0,671 & 0,627 & 0,619 & 0,606 & 0,595 & 0,615 \\
\hline$\omega=h \cdot M / P$ & & 0,117 & 0,117 & 0,115 & 0,114 & 0,113 & 0,113 & 0,110 & 0,109 & 0,109 & 0,110 \\
\hline$q=\omega /(\beta \cdot(1-\omega))$ & & 0,201 & 0,238 & 0,215 & 0,189 & 0,189 & 0,203 & 0,200 & 0,202 & 0,206 & 0,200 \\
\hline \multirow{2}{*}{$I C A F=g \cdot e / q / 4$} & тыс. руб. & 151,6 & 232,8 & 471,3 & 657,1 & 794,1 & 862,8 & 922,7 & 1023,9 & 1168,5 & 1314,0 \\
\hline & тыс. \$ & 527,2 & 480,8 & 583,5 & 764,3 & 803,2 & 540,6 & 463,8 & 530,1 & 573,3 & 628,0 \\
\hline
\end{tabular}

Примечание: значения, приведенные в рублях до 2015, надо умножать на 10000 взвези с деноминацией

ICAF, TbIC. \$

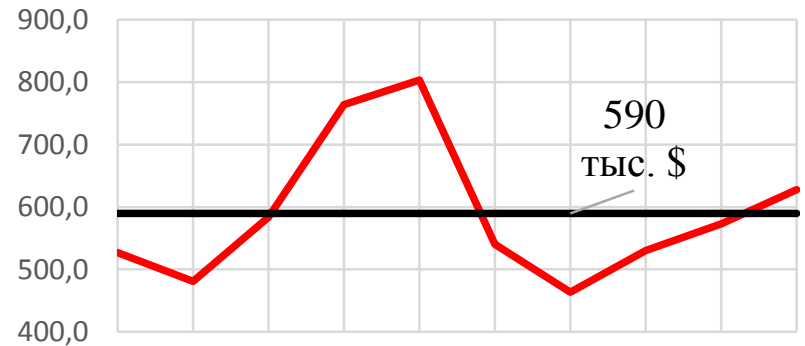

2010201120122013201420152016201720182019

Рисунок 3 - Затраты, которые экономически целесообразно инвестировать в сохранение жизни (ICAF) в РБ в 2010-2019 гг.

\section{Органичнее целевой функции}

С социальной точки зрения, риск отказа несущих конструкций зданий будет достаточно низким только тогда, когда он не влечет за собой уменьшения индекса качества жизни, рассчитываемого по формуле [13]:

$$
L Q I=g^{q} \cdot e
$$

где $g, q, e$ - тоже, что и в (21).

Изменения значении LQI для экономических условий Республики Беларусь полученное на основании статистических справочников [10-12], приведено на рисунке 4.

\section{LQI, \$год}

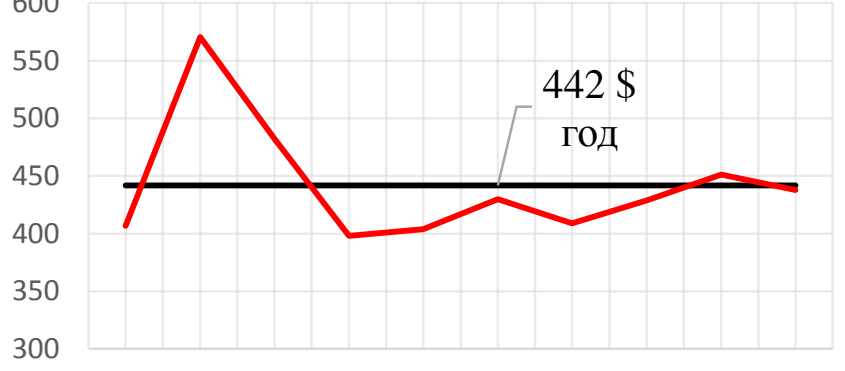

2010201120122013201420152016201720182019

Рисунок 4 - Индекс качества жизни (LQI) в РБ в 2010-2019 гг.

Функция (21) не будет убывать, если значение ее производной будет не ниже нуля. Так как заложенный в проектное решение индекс безопасности оказывает влияние на значение внутреннего валового продукта на душу населения $(g)$ и на среднюю ожидаемую продолжительность жизни (e), возьмем производную по этим переменным и запишем:

$$
\begin{gathered}
d g / g+d e /(q \cdot e) \geq 0 \\
\text { или }-d g \geq g / q \cdot d e / e \approx g / q \cdot C_{x} \cdot d \mu,
\end{gathered}
$$

где $d g$ - придельное изменение ВВП на душу населения;

$d \mu$ - предельное снижение смертности;

$C_{x}$ - демографическая константа, представляющая собой остаточную продолжительность жизни среднестатистического индивида. 
Вестник Брестского государственного технического университета. 2021

Значение демографической константы $\left(C_{x}\right)$ для Республики Беларусь было рассчитано по методике, изложенной в [22], с использованием данных, представленных в демографических справочниках [14-20]. В период с 2011 по 2019 года это значение находилось в пределах 34,4-35,7 лет.

После умножения на численность населения неравенство (23) примет вид:

$$
\begin{aligned}
& d g \cdot N_{p} \geq g / q \cdot d e / e \cdot N_{p} \\
& \text { или } d c \geq-g / q \cdot C_{x} \cdot d m,
\end{aligned}
$$

где $d c$ - предельные издержки для общества, которые на уровне проекта следует определять как прямые инвестиции в безопасность жизни;

$d m$ - предельное снижение риска для жизни, определяемое как снижение вероятности отказа конструкции $\left(d P_{f}\right)$, умноженное на ожидаемое число смертельных исходов в случае его наступления $\left(N_{F}\right)$

Выразим из неравенства (24) предельное снижение риска для жизни. Отметим, что обе части полученного неравенства зависят от параметра принятия решения (р), под которым понимается отношение среднего значения эффекта сопротивления к среднему значению эффекта воздействия:

$$
-d m(p) \leq d c(p) \cdot q /\left(g \cdot C_{x}\right)=d c(p) / S W T P,
$$

где SWTP - показатель сохранения жизни.

Рассмотрим неравенство (25) как ограничение для целевой функции (10). Варьируя параметры, приведенные в таблице 2, построим графики зависимости значения целевой функции (общих затрат), затрат на безопасность, затрат, связанных с отказом, а также риски для жизни от параметра принятия решения (рис. 5).

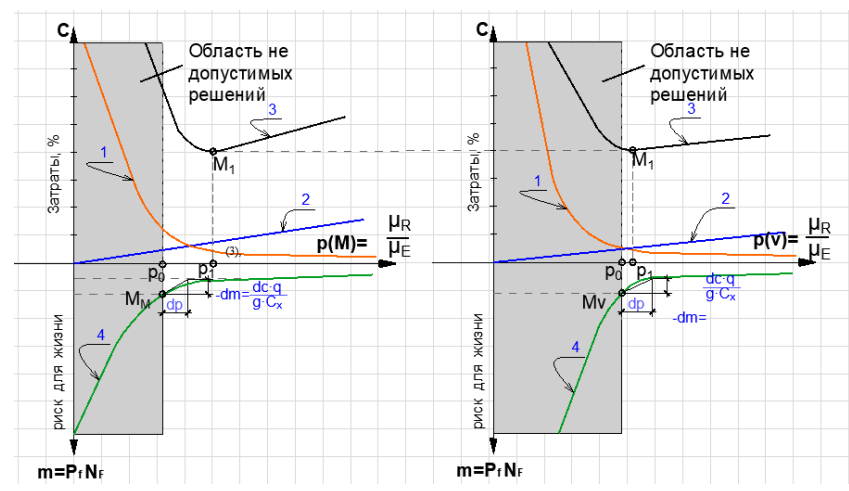

1 - затраты, связанные с отказом; 2 - затраты на безопасность;

3 - целевая функция (общие затраты); 4 - риск для жизни

Рисунок 5 - Определение оптимального значения параметров принятия решения $p(M)$ при изгибе и $p(v)$ при продавливании

На рисунке 5 приведен график целевой функции (3), включающей в себя все затраты на протяжении всего срока службы конструкции (в т. ч. затраты на безопасность (2) и затраты, связанные с отказом конструкции (1)). Минимумом данной фуннкции является точка $M_{1}$. Также на рисунке 5 приведен график (4) зависимости риска для жизни от параметров принятия решения $p(M)$ и $p(v)$. Точки $M_{M}$ и $M_{V}$ графрика (4) соответствуют таким значениям параметров принятия решения, при которых предельное изменение риска для жизни соответствует отношению инвестиций в безопасность к показателю сохранения жизни. Область на графике левее точек $M_{M}$ и $M v$ заштрихована. Это область советует проектным решением с высоким, социально неприемлемым риском для жизни людей. Незаштрихованная область, расположенная правее точки точек $M_{M}$ и $M_{V}$, соответствует области допустимых решений задачи оптимизации.

Данные графики построены для конструктивных систем с плоскими плитами перекрытий, с шагом колонн 6 м, загруженной собственным весом и нагрузкой с нормативным значением $4 \mathrm{kH} / \mathrm{m}^{2}$. Точка $M_{1}$ в рассмотренном примере лежит правее точек $M_{M}$ и $M v$, т. е. в области допустимых решений целевой функции. Поэтому конструкция с проектными параметрами, соответствующими точки $M_{1}$, является решением задачи оптимизации, а индекс надежности этой конструкции является экономически оптимальным и социально приемлемым.

При выборе наиболее подходящего метода оптимизации для решения поставленной задачи следует учесть, что область допустимых значений варьируемых параметров объемная (составляет 2470 возможных вариантов), и то, что для определения вероятности отказа используется громоздкий в вычислительном плане метод (симуляция Монте-Карло). Перечисленные обстоятельства ограничивают применения метода последовательного перебора вариантов и значительно снижают точность методов случайного поиска. Также следует отметить, что варьируемые параметры связаны друг с другом (например, возрастание толщины перекрытия при равной вероятности отказа ведет к снижению процента армирования плиты и наоборот). Это указывает на наличие оврагов в рельефе целевой функции, что исключает использование метода покоординатного спуска. Выходом будет применение комбинационного метода, сочетающего в себе метод случайного поиска и метод поиска по деформированному многоугольнику, который хорошо зарекомендовал себя при поиске экстремумов функций с линейными и нелинейными оврагами.

Описанная методика была применена для оптимизации 15 проектных решений перекрытий. Результаты оптимизации: оптимальные значений целевых индексов надежности и проектных парамет-

\begin{tabular}{|c|c|c|c|c|c|}
\hline $\begin{array}{c}\text { Пролет } \\
\text { перекрытия - L, м }\end{array}$ & 4 & 5 & 6 & 7 & 8 \\
\hline \multicolumn{6}{|c|}{$\mathrm{Q}=2 \mathrm{kH} / \mathrm{M}^{2} \mathrm{Gn}=2 \mathrm{kH} / \mathrm{M}^{2}$} \\
\hline $\begin{array}{c}\text { Толщина } \\
\text { перекрытия - } h \text {, см }\end{array}$ & 0,16 & 0,18 & 0,2 & 0,24 & 0,3 \\
\hline $\begin{array}{l}\text { Ширина сечения } \\
\text { колонны - } b, \text { см }\end{array}$ & 0,3 & 0,4 & 0,55 & 0,6 & 0,6 \\
\hline $\begin{array}{c}\text { Коэфффициент } \\
\text { армирования } \rho, \%\end{array}$ & 0,25 & 0,35 & 0,4 & 0,45 & 0,55 \\
\hline $\begin{array}{c}\text { Вероятность } \\
\text { отказа } P_{f}\end{array}$ & $4,56 \mathrm{E}-08$ & $4,10 \mathrm{E}-07$ & $1,94 \mathrm{E}-06$ & 2,94E-06 & 5,21E-06 \\
\hline $\begin{array}{c}\text { Индекс } \\
\text { надежности } \beta\end{array}$ & 5,3 & 4,9 & 4,6 & 4,5 & 4,4 \\
\hline \multicolumn{6}{|c|}{$Q=2 \kappa H / M^{2} G n=4 \kappa H / M^{2}$} \\
\hline $\begin{array}{c}\text { Толщина } \\
\text { перекрытия - } h \text {, см }\end{array}$ & 0,18 & 0,22 & 0,24 & 0,26 & 0,32 \\
\hline $\begin{array}{l}\text { Ширина сечения } \\
\text { колонны - } b, \text { см }\end{array}$ & 0,3 & 0,4 & 0,55 & 0,7 & 0,75 \\
\hline $\begin{array}{c}\text { Коэфффициент } \\
\text { армирования } \rho, \%\end{array}$ & 0,3 & 0,35 & 0,4 & 0,55 & 0,6 \\
\hline $\begin{array}{c}\text { Вероятность } \\
\text { отказа } P_{f}\end{array}$ & $1,56 \mathrm{E}-08$ & $1,278 \mathrm{E}-07$ & 2,90E-07 & 6,08E-07 & 1,91E-06 \\
\hline $\begin{array}{c}\text { Индекс } \\
\text { надежности } \beta\end{array}$ & 5,5 & 5,2 & 5,0 & 4,9 & 4,6 \\
\hline \multicolumn{6}{|c|}{$Q=2 \kappa H / M^{2} G n=6 \kappa H / M^{2}$} \\
\hline $\begin{array}{c}\text { Толщина } \\
\text { перекрытия }-h, \text { см }\end{array}$ & 0,2 & 0,24 & 0,28 & 0,32 & 0,36 \\
\hline $\begin{array}{l}\text { Ширина сечения } \\
\text { колонны - } b, \text { см }\end{array}$ & 0,3 & 0,4 & 0,6 & 0,75 & 0,75 \\
\hline $\begin{array}{c}\text { Коэфффициент } \\
\text { армирования } \rho, \%\end{array}$ & 0,3 & 0,35 & 0,55 & 0,65 & 1 \\
\hline $\begin{array}{c}\text { Вероятность } \\
\text { отказа } \mathrm{P}_{\mathrm{f}}\end{array}$ & 1,31E-08 & 5,54E-08 & 1,17E-07 & $2,75 E-07$ & 4,02E-07 \\
\hline $\begin{array}{c}\text { Индекс } \\
\text { надежности } \beta\end{array}$ & 5,6 & 5,3 & 5,2 & 5,0 & 4,9 \\
\hline
\end{tabular}
ров приведены в таблице 5 .

Таблица 5 - Результаты оптимизации 


\section{Выводы}

Для каждой конструкции существует свой оптимальный индекс надежности, в соответствии с которым ее и следует проектировать. Значение индекса надежности конструкции с плоскими плитами перекрытия варьируется от 5,6 до 4,4 в зависимости от шага колонн и нагрузок на перекрытия.

Оптимальный индекс надежности конструкции следует определять в соответствии с методами оптимального проектирования В качестве целевой функции для конструкций с плоскими плитами следует рассматривать общую стоимость плит перекрытия и колонн,

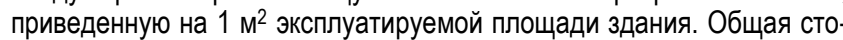
имости конструкций определяется на протяжении всего срока службы, с учетом ликвидации последствий ее вероятностного отказа. Она включает в себя стоимость возведения конструкции, затраты, обусловленные износом, и затраты, связанные с ликвидацией последствий вероятностного отказа, определяемые с учетом денежной суммы, которую экономически целесообразно инвестировать в сохранение жизни

В качестве ограничения целевой функции следует рассматривать ограничения риска для жизни с социальной точки зрения. Вероятность отказа конструкции должна быть настолько низкой, чтобь не вызывать изменения индекса качества жизни (LQI) в меньшую сторону.

Для расчета индекса качества жизни необходимо определить: коэффициент, зависящий от продолжительности активной фазь жизни $(q)$, соотношение труда и отдыха населения $(\omega)$, и демографическую константу $\left(C_{x}\right)$, представляющую собой остаточный срок жизни среднестатистического индивида в Республики Беларусь. Эти параметры были рассчитаны по существующим методикам на основании статистических данных. В 2019 их значения составляли: $\omega=0,24 \cdot 0,46=0,11 ; q=0,11 /(0,615 \cdot(1-0,11))=0,2 ; C_{x}=35,7$ лет. Они могут быть использовании при оптимизации надежности других конструкций.

\section{Список цитированных источников}

1. Klein, B. Further remarks on the direct use of extremal principles in solving certain optimizing problem involving inequalities / B. Klein // Journal of the Operations Research Society of America. - 1955. № 3. - Р. 548-554.

2. Основы проектирования строительных конструкций [Электронный ресурс]: СН 2.01.01-2019. - Введ. 08.09.2020. Режим доступа https://ips3.belgiss.by/TnpaDetail.php?Urlld=624926. - Дата доступа : 03.09.2020.

3. Мартынов, Ю. С. Вероятностные модели воздействий для условий Республики Беларусь / Ю. С. Мартынов, В.В.Надольский // Вестник полоцкого государственного университета. Строительство. Прикладные науки. Строительные конструкции. - 2014. № 16 - C. 13-19.

4. JCSS-Joint Committee on Structural Safety [Electronic resource] // Probabilistic Model Code. 2001. - Mode of access: http:// www.jcss.ethz.ch/. - Date of access: 21.03.2017.

5. Бетон. Часть 1. Требования, показатели, изготовление и соответствие : СТБ ЕN 206-1-2009. - Введ. 01.01.10 ; срок действия 01.06.12 ; заменен СТБ EN 206-1-2011. - Минск : Стройтехнорм, 2009. - $70 \mathrm{c}$

6. Арматура для железобетонных конструкций. Арматура сварная. Общие технические условия : СТБ ЕN 10080-2011. - Взамен СТБ EN 10080-2009 ; введ. 01.06.12. - Минск : Стройтехнорм, 2012. $-60 \mathrm{c}$

7. Надежность строительных конструкций. Общие принципы : CTE ISO 2394-2015. - Введ. 01.01.2016. - Минск : Госстандарт, 2016. $-69 \mathrm{c}$.

8. Инструкция о порядке определения сметной стоимости строительства и составления сметной документации на основании нормативов расхода ресурсов в натуральном выражении : Постановление Минстройархитектуры Республики Беларусь от 18.11.2011 № 51 // Национальный реестр правовых актов Республики Беларусь. - 2011. - № 144. - 8/24543.

9. On the assessment of marginal life saving costs for risk acceptance criteria [Electronic resource] / Katharina Fischer, Edgar Virguez, Mauricio Sanches-Silva, Michael H. Faber // Structural Safety. -
2013. - Journal homepage. - Mode of access: http:// www.elsevier.com/locate/strusafe. - Data of access: 27.08.2020.

10. Беларусь в цифрах: статистический справочник / Национальный статистический комитет Республики Беларусь ; редкол.: В. И. Зиновский [и др.]. - Минск : Национальный статистический комитет Республики Беларусь, 2012. - 100 с.

11. Беларусь в цифрах: статистический справочник / Национальный статистический комитет Республики Беларусь ; редкол.: И. В. Медведева [и др.]. - Минск : Национальный статистический комитет Республики Беларусь, 2016. - 72 с.

12. Беларусь в цифрах: статистический справочник / Национальный статистический комитет Республики Беларусь ; редкол.: И. В. Медведева [и др.]. - Минск : Национальный статистический комитет Республики Беларусь, 2020. - 72 с.

13. Жилые здания [Электронный ресурс]: CH 3.02.01-2019. - Введ. 08.09.2020 // Режим доступа : https://ips3.belgiss.by/TnpaDetail.php?Urlld=624915. - Дата доступа : 02.09.2020.

14. Демографический ежегодник Республики Беларусь: статистический сборник / Национальный статистический комитет Республики Беларусь ; редкол.: В. И. Зиновский [и др.]. - Минск : Национальный статистический комитет Республики Беларусь, 2013. $420 \mathrm{c}$.

15. Демографический ежегодник Республики Беларусь: статистический сборник / Национальный статистический комитет Республики Беларусь ; редкол.: В. И. Зиновский [и др.]. - Минск : Национальный статистический комитет Республики Беларусь, 2014. $414 \mathrm{c}$.

16. Демографический ежегодник Республики Беларусь: статистический сборник / Национальный статистический комитет Республики Беларусь ; редкол.: И. В. Медведева [и др.]. - Минск : Национальный статистический комитет Республики Беларусь, 2015. 449 c.

17. Демографический ежегодник Республики Беларусь: статистический сборник / Национальный статистический комитет Республики Беларусь ; редкол.: И. В. Медведева [и др.]. - Минск : Национальный статистический комитет Республики Беларусь, 2016. 442 c.

18. Демографический ежегодник Республики Беларусь: статистический сборник / Национальный статистический комитет Республики Беларусь ; редкол.: И. В. Медведева [и др.]. - Минск : Национальный статистический комитет Республики Беларусь, 2017. $440 \mathrm{c}$.

19. Демографический ежегодник Республики Беларусь: статистический сборник / Национальный статистический комитет Республики Беларусь ; редкол.: И. В. Медведева [и др.]. - Минск : Национальный статистический комитет Республики Беларусь, 2018. $431 \mathrm{c}$.

20. Демографический ежегодник Республики Беларусь: статистический сборник / Национальный статистический комитет Республики Беларусь ; редкол.: И. В. Медведева [и др.]. - Минск : Национальный статистический комитет Республики Беларусь, 2019. 429 c.

21. Жилые здания ГЭлектронный ресурс]: CH 3.02.01-2019. - Введ. 08.09.2020 // Режим доступа : https://ips3.belgiss.by/TnpaDetail.php?Urlld=624915. - Дата доступа : 02.09.2020.

22. Беларусь - валовой внутренний продукт [Электронный ресурс] / / Мировой атлас данных . Беларусь. Экономика. - Режим доступа : https://knoema.ru/atlas/Беларусь/ВВП. - Дата доступа : 02.09.2019.

23. Nathwani JS, Lind NC, Pandey MD. Affordable safety by choice: the life quality method. - Waterloo : University of Waterloo,1997.

24. Life tables for 191 countries: data, methods and results criteria [Electronic resource] / Alan D Lopez, Joshua Salomon, Omar Ahmad, Christopher JL Murray, Doris Mafat // World Health Organization: GPE Discussion Paper Series: No.9. - Mode of access: https://www.who.int/healthinfo/paper09.pdf. - Data of access: 27.08.2020.

25. Демографический ежегодник Республики Беларусь: статистический сборник / Национальный статистический комитет Республики Беларусь ; редкол.: Е. И. Кухаревич [и др.]. - Минск : Национальный статистический комитет Республики Беларусь, 2012. 501 c. 


\section{References}

1. Klein, V. Further remarks on the direct use of extremal principles in solving certain optimizing problem involving inequalities / B. Klein // Journal of the Operations Research Society of America. - 1955. № 3. - P. 548-554.

2. Osnovy proektirovaniya stroitel'nyh konstruk-cij [Elektronnyj resurs] SN 2.01.01-2019. - Vved. 08.09.2020. Rezhim dostupa https://ips3.belgiss.by/TnpaDetail.php?Urlld=624926. - Data dostupa : 03.09.2020.

3. Martynov, YU. C. Veroyatnostnye modeli vozdejstvij dlya uslovij Respubliki Belarus' / YU. S. Martynov, V. V. Nadol'skij // Vestnik polockogo gosudarstvennogo universiteta. Stroitel'stvo. Prikladnye nauki. Stroitel'nye konstrukcii. - 2014. - № 16 - S. 13-19.

4. JCSS-Joint Committee on Structural Safety [Electronic resource] // Probabilistic Model Code. 2001. - Mode of access: http://www.jcss.ethz.ch/. - Date of access: 21.03.2017.

5. Beton. CHast' 1. Trebovaniya, pokazateli, izgotovlenie i sootvetstvie : STB EN 206-1-2009. - Vved. 01.01.10 ; srok dejstviya 01.06.12; zamenen STB EN 206-1-2011. - Minsk : Strojtekhnorm, 2009. - $70 \mathrm{~s}$.

6. Armatura dlya zhelezobetonnyh konstrukcij. Armatura svarnaya. Obshchie tekhnicheskie usloviya : STB EN 10080-2011. - Vzamen STB EN 10080-2009 ; vved. 01.06.12. - Minsk : Strojtekhnorm, 2012. $-60 \mathrm{~s}$

7. Nadezhnost' stroitel'nyh konstrukcij. Obshchie principy : STB ISO 2394-2015. - Vved. 01.01.2016. - Minsk : Gosstandart, 2016. - 69 s.

8. Instrukciya o poryadke opredeleniya smetnoj stoimosti stroi-tel'stva sostavleniya smetnoj dokumentacii na osnovanii normativov raskhoda resursov v natural'nom vyrazhenii : Po-stanovlenie Minstrojarhitektury Respubliki Belarus' ot 18.11.2011 № 51 // Nacional'nyj reestr pravovyh aktov Respubliki Belarus'. - 2011. - № 144. - 8/24543.

9. On the assessment of marginal life saving costs for risk acceptance criteria [Electronic resource] / Katharina Fischer, Edgar Virguez, Mauricio Sanches-Silva, Michael H. Faber // Structural Safety. - 2013. Journal homepage. - Mode of access: http://www.elsevier.com/locate/strusafe. - Data of access: 27.08.2020.

10. Belarus' v cifrah: statisticheskij spravochnik / Nacional'nyj statisticheskij komitet Respubliki Belarus' ; redkol.: V. I. Zinovskij [i dr.]. Minsk : Nacional'nyj statisticheskij komitet Respubliki Belarus', 2012. $100 \mathrm{c}$.

11. Belarus' v cifrah: statisticheskij spravochnik / Nacional'nyj statisticheski] komitet Respubliki Belarus' ; redkol.: I. V. Medvedeva [i dr.]. - Minsk Nacional'nyj statisticheskij komitet Respubliki Belarus', 2016. - 72 c.

12. Belarus' v cifrah: statisticheskij spravochnik / Nacional'nyj statisticheskij komitet Respubliki Belarus' ; redkol.: I. V. Medvedeva [i dr.]. Minsk : Nacional'nyj statisticheskij komitet Respubliki Belarus', 2020. $72 \mathrm{c}$.

13. ZHilye zdaniya [Elektronnyj resurs]: SN 3.02.01-2019. - Vved. 08.09.2020 // Rezhim dostupa : https://ips3.belgiss.by/TnpaDetail.php?Urlld=624915. - Data dostupa : 02.09.2020.
14. Demograficheskij ezhegodnik Respubliki Belarus': statisticheskij sbornik / Nacional'nyj statisticheskij komitet Respubliki Belarus' ; redkol.: V. I. Zinovskij [i dr.]. - Minsk : Nacional'nyj statisticheskij komitet Respubliki Belarus', 2013. - $420 \mathrm{c}$.

15. Demograficheskij ezhegodnik Respubliki Belarus': statisticheskij sbornik / Nacional'nyj statisticheskij komitet Respubliki Belarus' ; redkol.: V. I. Zinovskij [i dr.]. - Minsk : Nacional'nyj statisticheski] komitet Respubliki Belarus', 2014. - $414 \mathrm{c}$.

16. Demograficheskij ezhegodnik Respubliki Belarus': statisticheskij sbornik / Nacional'nyj statisticheskij komitet Respubliki Belarus' ; redkol.: I. V. Medvedeva [i dr.]. - Minsk : Nacional'nyj statisticheski] komitet Respubliki Belarus', 2015. - 449 c.

17. Demograficheskij ezhegodnik Respubliki Belarus': statisticheskij sbornik / Nacional'nyj statisticheskij komitet Respubliki Belarus' ; redkol.: I. V. Medvedeva [i dr.]. - Minsk : Nacional'nyj statisticheski] komitet Respubliki Belarus', 2016. - 442 c.

18. Demograficheskij ezhegodnik Respubliki Belarus': statisticheskij sbornik / Nacional'nyj statisticheskij komitet Respubliki Belarus' ; redkol.: I. V. Medvedeva [i dr.]. - Minsk : Nacional'nyj statisticheski] komitet Respubliki Belarus', 2017. - $440 \mathrm{c}$.

19. Demograficheskij ezhegodnik Respubliki Belarus': statisticheskij sbornik / Nacional'nyj statisticheskij komitet Respubliki Belarus' ; redkol.: I. V. Medvedeva [i dr.]. - Minsk : Nacional'nyj statisticheski] komitet Respubliki Belarus', 2018. - $431 \mathrm{c}$.

20. Demograficheskij ezhegodnik Respubliki Belarus': statisticheski sbornik / Nacional'nyj statisticheskij komitet Respubliki Belarus' ; redkol.: I. V. Medvedeva [i dr.]. - Minsk : Nacional'nyj statisticheski] komitet Respubliki Belarus', 2019. - $429 \mathrm{c}$.

21. ZHilye zdaniya [Elektronnyj resurs]: SN 3.02.01-2019. - Vved 08.09.2020 // Rezhim dostupa : https://ips3.belgiss.by/TnpaDetail.php?Urlld=624915. - Data dostupa : 02.09.2020.

22. Belarus' - valovoj vnutrennij produkt [Elektronnyj resurs] / / Mirovoj atlas dannyh. Belarus'. Ekonomika. - Rezhim dostupa https://knoema.ru/atlas/Belarus'/VVP. - Data dostupa : 02.09.2019.

23. Nathwani JS, Lind NC, Pandey MD. Affordable safety by choice: the life quality method. - Waterloo : University of Waterloo,1997.

24. Life tables for 191 countries: data, methods and results criteria [Electronic resource] / Alan D Lopez, Joshua Salomon, Omar Ahmad, Christopher JL Murray, Doris Mafat // World Health Organization: GPE Discussion Paper Series: No.9. - Mode of access: https://www.who.int/healthinfo/paper09.pdf. - Data of access: 27.08.2020.

25. Demograficheskij ezhegodnik Respubliki Belarus': statisticheskij sbornik / Nacional'nyj statisticheskij komitet Respubliki Belarus' ; redkol.: E. I. Kuharevich [i dr.]. - Minsk : Nacional'nyj statisticheski] komitet Respubliki Belarus', 2012. - 501 c.

Материал поступил в редакцию 27.05.2021 